\title{
A NOVEL SUCLA2 MUTATION IN A PORTUGUESE PATIENT
}

Nogueira C ${ }^{1}$, Garcia P ${ }^{2}$, Diogo L 2 , Valongo C ${ }^{1}$, Santorelli FM ${ }^{3}$, Vilarinho $L^{1}$

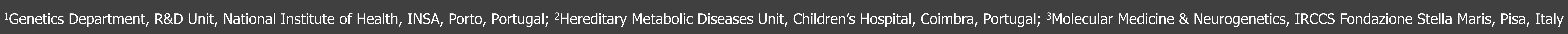

\section{INTRODUCTION}

Succinyl COA synthase is a mitochondrial matrix enzyme that catalyzes the reversible synthesis of succinate and ATP or GTP from succinyl-CoA and ADP in the tricarboxylic acid cycle (TCA). This enzyme is made up of two subunits, a and $\beta$, encoded by SUCLG1 and SUCLA2, respectively (Figure 1) [1].

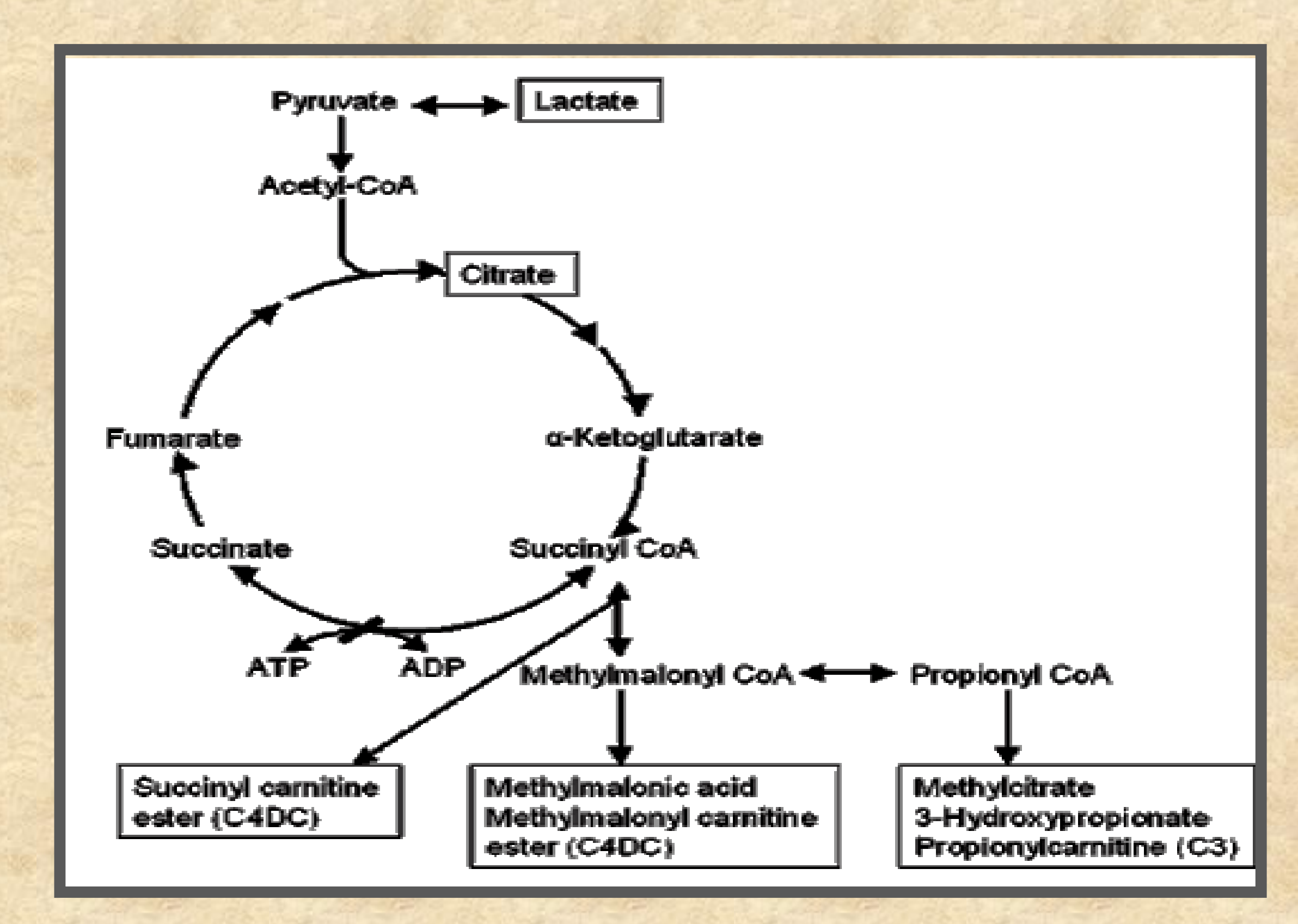

Figure 1. Relevant metabolic pathways illustrating the metabolic effects of ADP-forming succinyl-CoA synthetase deficiency.

The clinical features of patients with mutations in SUCLA2 include early childhood hypotonia, developmental delay, and almost invariably, progressive dystonia and sensorineural deafness. Mutations in SUCLA2 and SUCLG1 cause an encephalomyopathic form of infantile mtDNA depletion syndrome[2].

A useful diagnostic clue in succinyl CoA synthase disorders is a "mildly" elevated urinary methylmalonic acid (MMA), and presence of TCA intermediates.

To date, few patients with SUCLG1 mutations have been reported, whereas mutations in SUCLA2 have been reported in 20 patients [3]. We here present an additional patient with a novel SUCLA2 mutation.

\section{PATIENTS AND METHODS}

We report a 17-month-old-boy, who presented severe muscular hypotonia, failure to thrive, developmental delay, weight loss during a gastroenteritis crises, dysmorphisms and muscular atrophy.

A biochemical investigation disclosed hyperlactacidemia together with moderate excretion of MMA and elevated C4-dicarboxylic carnitine (C4DC).

Sequencing analysis of SUCLA2 and SUCLG1, mitochondrial DNA quantification and western blot was performed in patient's fibroblasts, using standard methods.

\section{RESULTS}

Mutation analysis of SUCLA2 revealed a novel homozygous c.985A>G mutation in exon 8 (p.M329V) (Figure 2A). The involvement of SUCLA2 was confirmed by western blot analysis, which showed some residual amount of SUCLA2 protein in patient's fibroblasts (Figure 2B). The patient also presented a moderate mitochondrial DNA depletion in fibroblasts $(40 \%$ of aged-matched controls). This missense mutation affects an amino acid that is highly conserved in different species and was not found in controls. The analysis by bioinformatics tools also confirmed a pathogenic mutation (Figure 3). Altogether, these findings indicate that the identified mutation is pathogenic and responsible for this disorder.

A

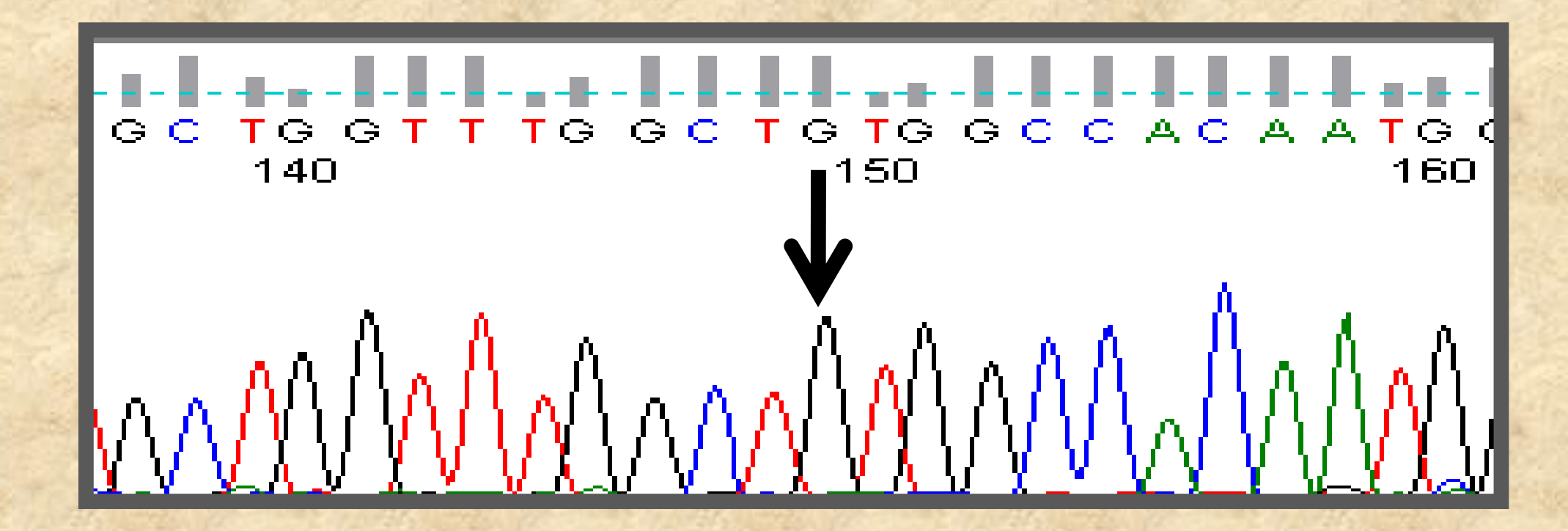

Figure 2. Identification of a novel SUCLA2 mutation. A) Patient's SUCLA2 sequence with the p.M329V mutation; B) Western-blot analysis of patient's fibroblasts ( $p$ ) and a control subject (C).

A
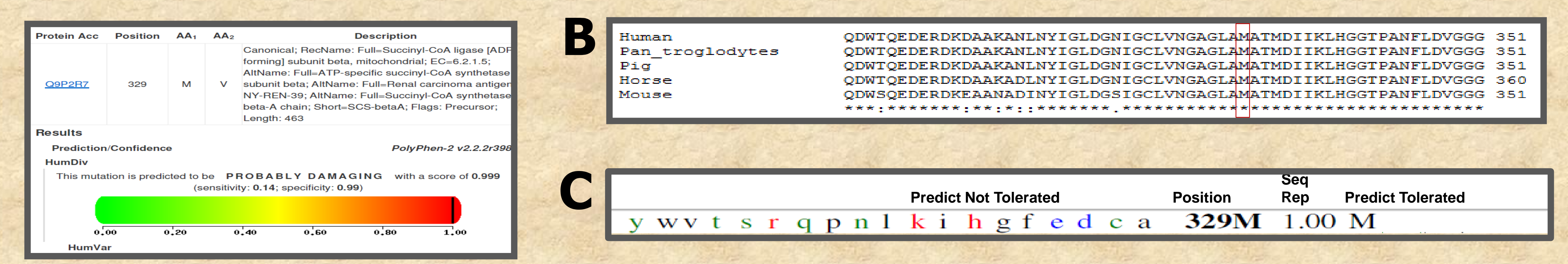

Figure 3. Analysis by bioinformatics tools of a SUCLA2 mutation: p.M329V. A) PolyPhen-2 prediction of functional effects of human variations; B) ClustalW aligments; C) Sift predictions.

\section{DISCUSSION / CONCLUSION}

The clinical and biochemical phenotype of our patient is strikingly similar to other reported patients with SUCLA2 mutations [1]. In addition, the mildly elevated levels of MMA and C4DC raised the suspicion of this disease, which was confirmed by the identification of a novel mutation in SUCLA2. In agreement with this results, western blot showed some residual amount of SUCLA2 protein and a moderate reduction of mitochondrial DNA copy number was found in patient's fibroblasts.

Our study contributed to expand the spectrum of patients with SUCLA2 mutations, and will be important for an accurate genetic counseling and a prenatal diagnosis to the affected family.

\section{REFERENCES}

